\title{
$\mathrm{M}|\mathrm{R}| \mathrm{S}$ Internet Journal Nitride Semiconductor Research
}

\section{Review of Pendeo-Epitaxial Growth and Characterization of Thin Films of GaN and AlGaN Alloys on $6 \mathrm{H}-\mathrm{SiC}(0001)$ and $\mathrm{Si}(111)$ Substrates}

\author{
Robert F. Davis $^{1}$, T. Gehrke ${ }^{1}$, K.J. Linthicum ${ }^{1}$, P. Rajagopal ${ }^{1}$, A.M. Roskowski ${ }^{1}$, T. Zheleva ${ }^{1}$, \\ Edward A. Preble ${ }^{1}$, C.A. Zorman ${ }^{2}$, M. Mehregany ${ }^{2}$, U. Schwarz ${ }^{3}$, J. Schuck ${ }^{3}$ and R. Grober ${ }^{3}$ \\ ${ }^{1}$ Department of Materials Science and Engineering, North Carolina State University, \\ ${ }^{2}$ Department of Electrical, Systems and Computer Engineering, Case Western Reserve University, \\ ${ }^{3}$ Department of Applied Physics, Yale University,
}

(Received Monday, September 17, 2001; accepted Tuesday, November 20, 2001)

Discrete and coalesced monocrystalline $\mathrm{GaN}$ and $\mathrm{Al}_{\mathrm{x}} \mathrm{Ga}_{1-\mathrm{x}} \mathrm{N}$ layers grown via pendeo-epitaxy (PE) originated from side walls of $\mathrm{GaN}$ seed stripes with and without $\mathrm{SiN}_{\mathrm{X}}$ top masks have been grown via organometallic vapor phase deposition on GaN/AlN/6H-SiC(0001) and GaN(0001)/AlN(0001)/ 3C-SiC(111)/Si(111) substrates. Scanning and transmission electron microscopies were used to evaluate the external microstructures and the distribution of dislocations, respectively. The dislocation density in the laterally grown sidewall regions and in the regions grown over the $\mathrm{SiN}_{\mathrm{x}}$ masks was reduced by at least five orders of magnitude relative to the initial GaN seed layers. Tilting of $0.2^{\circ}$ in the coalesced GaN epilayers grown over the $\mathrm{SiN}_{\mathrm{X}}$ masks was determined via X-ray and selected area diffraction; however, tilting was not observed in the material suspended above the $\mathrm{SiC}$ substrate and that grown on unmasked stripes. A strong, low-temperature photoluminescence bandedge peak at $\sim 3.45 \mathrm{eV}$ with a FWHM of $<300 \mu \mathrm{eV}$ was determined on the overgrowth material grown on the silicon carbide substrates. The band-edge in the GaN grown on silicon substrates was shifted to a lower energy by $10 \mathrm{meV}$, indicative of a greater tensile stress.

\section{Introduction}

It has been a necessity for investigators in the III-nitride community to grow films of GaN and related nitride materials using heteroepitaxial routes because of the dearth of bulk substrates of these materials. This results in films containing dislocation densities of $10^{8}-10^{10} \mathrm{~cm}^{-}$

2 because of the mismatches in the lattice parameters and the coefficients of thermal expansion between the buffer layer and the film and/or the buffer layer and the substrate. These high concentrations of dislocations limit the performance of devices via carrier recombination, increased leakage currents, decreased breakdown strength and loss in efficiency and lifetime of optical devices.

Several groups [1] [2] [3] [4] [5] [6] [7] [8] [9] [10] [11], including the present authors, have conducted research regarding selective area growth (SAG) and lateral epitaxial overgrowth (LEO) techniques for $\mathrm{GaN}$ deposition, specifically to significantly reduce the dislo- cation density. These techniques have been employed previously to fabricate new microelectronic devices with better performance in Si [12], GaAs [13] and InP [14] semiconductor materials. The crystal quality of the overgrown layers of these materials was sufficiently good to be used for device applications. Increased emphasis in this research topic for III-Nitride materials was fueled in part by the announcement by Nakamura, et al. [15] [16] [17] of (1) the dramatic increase in projected lifetime of their GaN-based blue light-emitting laser diodes from a few hundred hours for diodes fabricated on conventionally grown layers to over 10,000 hours for diodes produced on LEO material and (2) the subsequent commercial introduction of the laser diodes. Using these approaches, researchers have been able to grow $\mathrm{GaN}$ films containing dislocation densities of $\approx 10^{5} \mathrm{~cm}^{-2}$ in the areas of overgrowth. However, to benefit from this reduction in defects, the placement of devices incorporating LEO technology is limited and confined to 
regions on the final GaN device layer that are located on the overgrown regions.

Recently we have pioneered a new approach to selective epitaxy of $\mathrm{GaN}$ and $\mathrm{Al}_{\mathrm{x}} \mathrm{Ga}_{1-\mathrm{x}} \mathrm{N}$ layers, namely, pendeo- (from the Latin: to hang or be suspended) epitaxy (PE) [18] [19] [20] [21] [22] [23] [24] [25] as a promising new process route leading to a single, continuous, large area layer; multilayer heterostructures; or discrete platforms of these materials. This novel thin film growth technique also alleviates the process alignment problems and the high and non-uniform residual stresses inherent in epilayers grown by the conventional LEO approach. It incorporates mechanisms of growth exploited by the conventional LEO process by using an amorphous mask to prevent vertical propagation of threading dislocations; however, it extends beyond the conventional LEO approach to employ the substrate itself as a pseudo-mask. This unconventional approach differs from LEO in that growth does not initiate through open windows on the (0001) surface of the GaN seed layer; instead, it is forced to selectively begin on the sidewalls of a tailored microstructure comprised of forms previously etched into this seed layer. Continuation of the pendeo-epitaxial growth of $\mathrm{GaN}$ or the growth of the $\mathrm{Al}_{\mathrm{x}} \mathrm{Ga}_{1-\mathrm{x}} \mathrm{N}$ layer until coalescence over and between these forms results in a complete layer of low defect-density $\mathrm{GaN}$ or $\mathrm{Al}_{\mathrm{x}} \mathrm{Ga}_{1-\mathrm{x}} \mathrm{N}$. This is accomplished in one $(\mathrm{GaN})$, two $\left(\mathrm{Al}_{\mathrm{x}} \mathrm{Ga}_{1-\mathrm{x}} \mathrm{N}\right)$ or multiple (multilayer heterostructure) re-growth steps. The need to align devices or masks for the growth of the subsequent layers over particular areas of overgrowth is eliminated, unless deposition only in certain areas is desired. A form of pendeo-epitaxy (maskless LEO) has recently been employed by the present authors [18] [20] [23] [24] [25] and by Nakamura et al. [26] to further optimize the performance of Nichia's newest laser diodes.

The following sections describe the experimental parameters necessary to achieve $\mathrm{GaN}$ and $\mathrm{Al}_{\mathrm{x}} \mathrm{Ga}_{1-\mathrm{x}} \mathrm{N}$ via PE on both masked and unmasked $\mathrm{SiC}(0001)$ and $\mathrm{Si}(111)$ substrates. The microstructural, structural and optical evidence obtained for the resulting films is also described, discussed and summarized.

\section{Experimental Procedures}

Each pendeo-epitaxial $\mathrm{GaN}$ and $\mathrm{Al}_{\mathrm{X}} \mathrm{Ga}_{1-\mathrm{x}} \mathrm{N}$ film and the underlying $\mathrm{GaN}$ seed layer and the AlN buffer layer were grown in a cold-wall, vertical pancake style RF inductively heated metallorganic vapor phase epitaxy (MOVPE) system. Two distinct process routes were explored for growth on (i) on-axis $6 \mathrm{H}-\mathrm{SiC}(0001)$ substrates and (ii) on-axis $\mathrm{Si}(111)$ substrates. In the former, each seed layer consisted of a $1 \mu \mathrm{m}$ thick GaN film deposited within the $980-1020^{\circ} \mathrm{C}$ range on a $100 \mathrm{~nm}$ thick AlN buffer layer previously deposited within the $980-1020^{\circ} \mathrm{C}$ range on an $6 \mathrm{H}-\mathrm{SiC}(0001)$ substrate. Triethylgallium, triethylaluminum and ammonia precursors were used in combination with a hydrogen diluent. Details of the experimental parameters used for the growth of these two layers are given in Ref. [27]. In the growth on the Si substrates, a $0.5-2.0 \mu \mathrm{m} 3 \mathrm{C}-\mathrm{SiC}(111)$ transition film was initially grown on a very thin $(\sim 20$ $\mathrm{nm}) 3 \mathrm{C}-\mathrm{SiC}(111)$ layer produced by conversion of the $\mathrm{Si}(111)$ surface at $1360^{\circ} \mathrm{C}$ for $90 \mathrm{~s}$ via reaction with a $15 \%$ propane $\left(\mathrm{C}_{3} \mathrm{H}_{8}\right) / 85 \% \mathrm{H}_{2}$.mixture. The transition film was subsequently achieved by simultaneously decreasing the flow rate of the $\mathrm{C}_{3} \mathrm{H}_{8} / \mathrm{H}_{2}$ mixture and introducing a $5 \% \mathrm{SiH}_{4} / 95 \% \mathrm{H}_{2}$ mixture. Both the conversion step and the $\mathrm{SiC}$ film deposition were achieved using a cold-wall, vertical geometry, RF inductively heated atmospheric pressure chemical vapor deposition (APCVD) reactor. Details of the experimental parameters used for the conversion step and the growth of the 3C-SiC layers are given in Ref. [28]. A $100 \mathrm{~nm}$ thick AlN buffer layer and a $0.5-1 \mu \mathrm{m} \mathrm{GaN}$ seed layer were subsequently deposited in the manner described above for the $6 \mathrm{H}-\mathrm{SiC}$ substrates.

Studies of the PE growth of the III-Nitrides were conducted using GaN seed layers on which had been initially deposited and patterned either (1) a $100 \mathrm{~nm}$ silicon nitride growth mask via plasma enhanced CVD that was covered by a nickel etch mask subsequently deposited using e-beam evaporation or (2) only the nickel mask. Patterning of the nickel mask was achieved using standard photolithography techniques. The final, tailored, microstructure consisting of $\mathrm{GaN}$ seed forms with or without the overlying silicon nitride mask (the latter will be subsequently referred to as maskless PE) was fabricated by removal via sputtering of portions of the nickel etch mask and by inductively coupled plasma (ICP) etching of portions of the silicon nitride growth mask, the GaN seed layer and the AlN buffer layer. The remaining areas of $\mathrm{Ni}$ mask protecting the seed structures during the ICP etching were removed from all samples using $\mathrm{HNO}_{3}$.

Critical to the success of the pendeo-epitaxial growth, the etching of the seed-forms was continued completely through the exposed $\mathrm{GaN}$ and AlN layers and into either the $6 \mathrm{H}-\mathrm{SiC}$ substrate or the $3 \mathrm{C}-\mathrm{SiC}$ layer, thereby removing all III- nitride material from the areas between the side walls of the forms. The seed forms used in this study were raised rectangular stripes oriented along the $\left[\begin{array}{lll}1 & 1 & 0\end{array} 0\right]$ direction, thereby providing a sequence of parallel GaN sidewalls (nominally $\left(\begin{array}{llll}1 & 1 & \overline{2} & 0\end{array}\right)$ faces). Seed form widths of 2 and $3 \mu \mathrm{ms}$ coupled with separation distances of 3 and $7 \mu \mathrm{ms}$ were employed. Immediately prior to pendeo-epitaxial growth, the pat- 
terned samples were dipped in an acid solution to remove surface contaminants from the walls of the underlying GaN seed structures.

A schematic of the pendeo-epitaxial growth of GaN is illustrated in Figure 1. There are three primary stages associated with the pendeo-epitaxial formation of this material: (i) initiation of lateral homoepitaxy from the sidewalls of the GaN seed, (ii) vertical growth and (iii) lateral growth over the silicon nitride mask covering the seed structure. Pendeo-epitaxial growth of $\mathrm{GaN}$ was achieved within the temperature range and pressure of $1050-1100^{\circ} \mathrm{C}$ and 40 torr, repsectively. Additional experimental details regarding the pendeo-epitaxial growth of $\mathrm{GaN}$ and $\mathrm{Al}_{\mathrm{x}} \mathrm{Ga}_{1-\mathrm{x}} \mathrm{N}$ layers employing $6 \mathrm{H}-$ $\mathrm{SiC}$ and Si substrates are given in Refs. [18] [19] [20] [21] [22] [23] [24] [25].

The morphology and defect microstructures were investigated using scanning electron microscopy (SEM) (JEOL $6400 \mathrm{FE}$ ) and transmission electron microscopy (TEM) (TOPCON 0002B, $200 \mathrm{KV}$ ). Determination of the degree of tilting in the $\mathrm{PE} \mathrm{GaN}$ was achieved using X-ray diffraction (XRD) (Philips X'Pert MRD X-ray diffractometer) and selected area diffraction TEM analysis. Optical characterization was performed via microphotoluminescence (PL) and micro-Raman with spatial resolution of $\sim 300 \mathrm{~nm}$ (PL) and $\sim 700 \mathrm{~nm}$ (Raman) using a He-Cd laser $(\lambda=325 \mathrm{~nm})$ and a confocal objective lense (Zeiss 65x).

\section{Results and Discussion}

\subsection{Growth from GaN and AIGaN Stripes Masked with Silicon Nitride}

\subsubsection{Silicon Carbide Substrates}

The pendeo-epitaxial phenomenon is made possible by taking advantage of growth mechanisms identified by Zheleva et al. [7] in the conventional LEO technique and by using two additional key steps, namely, the initiation of growth from a GaN face other than the (0001) and, as described in the Introduction, the use of the substrate (in this case a $\mathrm{SiC}$ wafer or a $\mathrm{SiC}$-coated $\mathrm{Si}$ ) as a pseudo-mask. By capping the seed-forms with a silicon nitride growth mask, the $\mathrm{GaN}$ is forced to grow initially on the AlN and the GaN sidewalls (PE event (i) in Figure 1). Common to conventional LEO, no growth occurs on the silicon nitride mask covering the seed forms. Deposition also does not occur on the exposed SiC surface areas at the higher growth temperatures employed to enhance lateral growth (the pseudo-mask effect). The Ga- and N-containing species more likely either diffuse along the surface or evaporate (rather than having sufficient time to form GaN nuclei) from both the silicon nitride mask and the silicon carbide substrate. The pronounced effect of this is shown in Figure 2 wherein the newly deposited GaN has grown truly suspended (pendeo) from the sidewalls of the GaN seed structure. During the second PE event ((ii) in Figure 1), vertical growth of GaN occurs from the advancing (0001) face of the laterally growing GaN. Once the vertical growth becomes extended to a height greater than the silicon nitride mask, the third PE event ((iii) in Figure 1) occurs, namely, conventional LEO-type growth and eventual coalescence over the seed structure, as shown in Figure 3 for an $\mathrm{Al}_{10} \mathrm{Ga}_{90} \mathrm{~N}$ film grown on a $\mathrm{PE} \mathrm{GaN}$ film.

Atomic force microscopy analysis of the (0001) surface revealed a typical step flow growth mode and a root mean square (rms) roughness of $0.48 \mathrm{~nm}$ for the over-

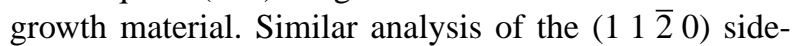
wall revealed an atomically smooth surface and a rms roughness of $0.096 \mathrm{~nm}$, where the steps were not resolved at the sensitivity of the instrument.

A cross-sectional TEM micrograph showing a typical pendeo-epitaxial growth structure over a silicon nitride masked stripe is shown in Figure 4. Threading dislocations extending into the $\mathrm{GaN}$ seed structure, originating from the GaN/AlN and $\mathrm{AlN} / \mathrm{SiC}$ interfaces are clearly visible. The silicon nitride mask acted as a barrier to the further vertical propagation of these defects into the laterally overgrown pendeo-epitaxial film. Since the newly deposited $\mathrm{GaN}$ is suspended above the $\mathrm{SiC}$ substrate, there are no vertically oriented defects associated with the mismatches in lattice parameters between GaN and AlN and between AlN and SiC. Preliminary analyses of the GaN seed/GaN PE and the AlN/GaN PE interfaces revealed evidence of the lateral propagation of the defects; however, there is yet no evidence that the defects reach the (0001) surface where device layers will be grown. As in the case of LEO, there is a significant reduction in the defect density in the regrown areas.

The continuation of the pendeo-epitaxial growth results in coalescence with adjacent growth fronts and the formation of a continuous layer of $\mathrm{GaN}$, as observed in cross-sectional and plan view SEM micrographs in Figures 5 (a) and (b), respectively. This also results in the practical elimination of all dislocations stemming from the heteroepitaxial growth of GaN/AIN on SiC. Clearly visible in Figure 5(a) are the voids that form when adjacent growth fronts coalesce. Optimization of the pendeo-epitaxial growth technique should eliminate these undesirable defects.

The low-temperature $(7 \mathrm{~K})$ photoluminescence spectrum of the coalesced PE GaN is shown in Figure 6. The edge emission band (3.454 eV), usually assigned to an exciton bound to a neutral donor $\left(\mathrm{X}-\mathrm{D}^{0}\right)$ is blueshifted $112 \mathrm{meV}$ compared to the GaN seed layer. The common weak yellow band was also observed for the pendeoepi- 
taxial layer. The blueshift is attributed to relaxation of the pendeoepitaxially grown $\mathrm{GaN}$.

\subsubsection{Silicon Substrates}

Research regarding development of process routes leading to the growth of III-Nitride films on Si has been minimal relative to the number of studies concerned with growth on sapphire and $\mathrm{SiC}$. This has been due in part to the three-dimensional nucleation and growth of $\mathrm{GaN}$ islands caused by the combination of significant mismatches in lattice parameters, the higher surface energy of $\mathrm{GaN}$ and the chemical reactivity of Si with the reactants in the growth environment. To address the above concerns we have developed a process route similar to those used for growth of $\mathrm{GaN}$ on $6 \mathrm{H}-\mathrm{SiC}(0001)$, but replaced the $6 \mathrm{H}-\mathrm{SiC}$ substrate with a $3 \mathrm{C}-\mathrm{SiC}(111)$ transition layer grown on a $\mathrm{Si}(111)$ substrate, in the manner discussed in Reference [28]. The atomic arrangement of the (111) plane of $3 \mathrm{C}-\mathrm{SiC}$ is equivalent to the (0001) plane of $6 \mathrm{H}-\mathrm{SiC}$; this facilitates the sequential deposition of a high temperature $2 \mathrm{H}-\mathrm{AlN}(0001)$ buffer layer of sufficient quality for the GaN seed layer. As described for the use of the $\mathrm{SiC}$ substrates, the $3 \mathrm{C}-\mathrm{SiC}$ layer must be exposed via etching and the resulting seed forms either capped with a silicon nitride growth mask or left uncapped.

For the initial demonstrations of PE growth of GaN films on silicon, 0.5 and $2 \mu \mathrm{m}$ thick $3 \mathrm{C}$-SiC layers were deposited on $50 \mathrm{~mm}$ diameter, $250 \mu \mathrm{m}$ thick converted $\mathrm{Si}(111)$ substrates. However, the $\sim 0.5 \mu \mathrm{m} 3 \mathrm{C}$-SiC layer cracked on cooling and did not provide an adequate barrier to the reaction with the Si substrate. All subsequent research described below used the $\sim 2.0 \mu \mathrm{m}$ barrier layer.

Figure 7 shows a cross-sectional SEM micrograph of a PE GaN layer grown laterally and vertically from raised GaN stripes etched in a GaN/AlN/3C-SiC/ $\mathrm{Si}(111)$ substrate and over the silicon nitride mask atop each stripe. Tilting between adjacent growth fronts over the mask regions are commonly observed in coalesced $\mathrm{GaN}$ epilayers grown using the LEO technique. This phenomenon was also determined to be present in the PE GaN films grown on masked seed structures. The splitting of the XRD rocking curves for the (0002) reflection are indicative of the crystallographic tilt and are dependent on the crystal orientation, as shown in Figure 8. The XRD spectrum taken along the $\left[\begin{array}{llll}1 & 1 & 0 & 0\end{array}\right]$, parallel to the stripes, consisted of one peak as shown in

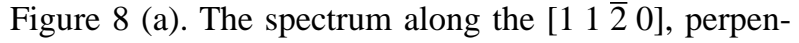
dicular to the stripes, exhibited two superimposed peaks, separated by a tilt of $0.2^{\circ}$, as shown in Figure 8 (b).

Unlike LEO, coalesced PE GaN epilayers obtained using silicon nitride masks contain two sets of coalesced growth fronts, namely, over the trenches and over the masks. A spatially sensitive method of evaluating the tilt in $\mathrm{PE}$ grown $\mathrm{GaN}$ is required to determine if it is present at both areas of coalescence. Figure 9 (a) shows a typical TEM selected area diffraction pattern taken from a small area of coalescence in a trench region. Figure 9 (b) shows the pattern taken near the region of coalescence over the silicon nitride mask. Analysis of these two patterns reveals little evidence of tilt in the laterally grown material over the trenches; however, significant tilt has occurred over the mask regions of the seed structures. The presence of two crystallographic orientations tilted towards each other have also been reported in $\mathrm{GaN}$ films grown via lateral epitaxial growth (LEO) [29] [30] [31] [32]. Thus the mask has markedly influenced the crystallographic tilt in the overgrown film. Additional influences of the masks on the quality of the overgrown GaN layer are shown (1) by the voids observed above the masks and similar to those shown in Figure 5 and Figure 7 and (2) the formation of boundaries in the area of coalescence due to the misregistry between the two growth fronts meeting above each mask, as shown in Figure 10. This last figure shows that the growth fronts on both sides of the coalescence boundary are highly defective as a result of the crystallographic misregistry at the boundary. Results similar to these have been reported for GaN films grown via LEO by Lilienthal-Weber, et al. [33]. These problems are also associated with the PE of $\mathrm{Al}_{\mathrm{x}} \mathrm{Ga}_{1-\mathrm{x}} \mathrm{N}$ films with the additional problem of nucleation of AlN on the masks.

A typical room temperature PL spectrum for a $\mathrm{PE}$ GaN film grown on $2 \mu \mathrm{m}$ wide raised stripes, oriented in the $\left[\begin{array}{llll}1 & \overline{1} & 0 & 0\end{array}\right]$ direction, spaced $3 \mu \mathrm{m}$ apart and capped with a silicon nitride mask is shown in Figure 11. The band-edge emission peak and a deep level (yellow luminescence) emission peak on a linear intensity scale were located at $363.6 \mathrm{~nm}(3.41 \mathrm{eV})$ and $\sim 570 \mathrm{~nm}$, respectively. The FWHM value of the band-edge emission was $109 \mathrm{meV}$. The low temperature (14K) PL spectrum on a logarithmic intensity scale showed a strong near bandedge emission at $359 \mathrm{~nm}(3.45 \mathrm{eV})$, as shown in Figure 12. The FWHM was $17 \mathrm{meV}$. Also included in Figure 12 is a low temperature PL spectrum of PE GaN grown on a GaN/AlN/6H-SiC substrate. The band-edge peak in the latter spectrum is at $358 \mathrm{~nm}(3.46 \mathrm{eV})$ with a FWHM of $16 \mathrm{meV}$. A comparison of these two spectra indicates that the optical character of the $\mathrm{PE} \mathrm{GaN} \mathrm{grown} \mathrm{on} \mathrm{the}$ $3 \mathrm{C}-\mathrm{SiC} / \mathrm{Si}$ substrate is nearly identical to the quality of the $\mathrm{PE} \mathrm{GaN}$ grown on the $6 \mathrm{H}-\mathrm{SiC}$ substrates. The bandedge of the $\mathrm{PE} \mathrm{GaN}$ grown on $\mathrm{Si}$ is shifted by $10 \mathrm{meV}$ to a slightly lower energy than that of the PE GaN grown on $\mathrm{SiC}$, which is indicative of a slightly higher tensile stress in the film of the former. This is caused by the larger lattice parameter of the Si substrate, compared to $\mathrm{SiC}$ and a bigger difference in thermal expansion coefficient between $\mathrm{GaN}$ and $\mathrm{Si}$. The linewidth of the near band-edge emission peak is increased in the case of the 
3C-SiC/Si substrate in comparison to the GaN/AlN/6H$\mathrm{SiC}$ substrate. This increase cannot be explained at this time.

\subsection{Growth from Unmasked GaN and AIGaN Stripes}

\subsubsection{Silicon Carbide Substrates}

The tilting and consequent void formation, crystallographic misregistry and boundary formation caused by growth over the silicon nitride masks shown by the $\mathrm{X}$ ray and TEM studies discussed above is unacceptable for a semiconductor film on which devices would be fabricated. The advantage of the masks in blocking the vertical propagation of threading dislocations from the initial $\mathrm{GaN}$ or $\mathrm{Al}_{\mathrm{x}} \mathrm{Ga}_{1-\mathrm{x}} \mathrm{N}$ stripes in the pendeo-epitaxial areas is outweighed by the formation of the boundaries and the horizontal dislocations due the crystallographic misregistry. As such, the use of masks was discontinued and a variation in the above process route was developed.

Electron beam lithography was applied to reduce the width of the GaN stripes to submicron dimensions, and the masking layer was eliminated. Figure 13(a) shows a GaN film grown from submicron wide, unmasked GaN seed posts on a $6 \mathrm{H}-\mathrm{SiC}$ substrate. Neither voids nor misregistry above the stripes is visible. The TEM micrograph in Figure 13(b) reveals a significant reduction in the density of vertically oriented threading dislocations in the laterally and vertically grown GaN relative to the stripes. Some dislocations threaded vertically, but with a gradual reduction in density. No dislocations or boundaries were generated within the areas of coalescence over the trench above the void. Selected area diffraction patterns from above the stripes and above the voids showed little evidence of tilt in this film.

Pendeo-epitaxial films of $\mathrm{Al}_{10} \mathrm{Ga}_{90} \mathrm{~N}$ were grown on submicron wide, unmasked $\mathrm{GaN}$ stripes on $6 \mathrm{H}-$ $\mathrm{SiC}(0001)$ substrates, as shown in Figure 14 (a). A large area scan via high resolution Auger electron spectroscopy revealed a variation in the Al content of $+/-0.5 \%$ [24]. The elimination of the masking layer was necessary, as $\mathrm{Al}_{\mathrm{x}} \mathrm{Ga}_{1-\mathrm{x}} \mathrm{N}$ nucleates on the silicon nitride mask layer. The TEM micrograph in Figure 14 (b) shows no lateral propagation of the vertically oriented threading dislocations in the laterally grown $\mathrm{Al}_{10} \mathrm{Ga}_{90} \mathrm{~N}$ regions. Only the threading dislocations within the GaN stripe propagated vertically into the newly deposited GaN. Selected area diffraction in the TEM of the areas of the film coalesced over the $\mathrm{Al}_{10} \mathrm{Ga}_{90} \mathrm{~N}$ stripes revealed neither tilting nor a boundary of coalescence, in contrast to those films that coalesced over the silicon nitride masks. The uniform density in the film contrast in Figure 14 (b) within the areas of coalesce over the $\mathrm{SiC}$ also indicates that no boundary formed in these regions. Dislocations were not observed within the areas of coalescence above the trench of this film. However, threading dislocations, normally stopped by the amorphous silicon nitride mask now extended into the newly deposited films. Preliminary analyses of the GaN seed/GaN PE and the AlN/ GaN PE interfaces revealed little evidence of the lateral propagation of these defects.

Spatially resolved strain and optical emission plots of stripe and wing regions were produced by microRaman and micro-PL measurements at a temperature of 8K. Micro-Raman measurements of an uncoalesced PE GaN Film indicate a shift in the $\mathrm{E}_{2}$ phonon to higher frequency in the wing with respect to the stripe (Figure 15). The shift corresponds to a relaxation in the wing material that is also measured in XRD by a reduction in the lattice parameters, $\mathrm{a}(-0.07 \%)$ and $\mathrm{c}(+0.03 \%)$ in the wing material. The wing material is relaxed with less strain and also emits very sharply in photoluminescence (PL). The donor bound exciton emission peak in the wing material has a FWHM value less than $300 \mu \mathrm{eV}$ (below the resolution of the equipment) compared to $12 \mathrm{meV}$ for the stripe material as seen in Figure 16. The sharp optical emission from the wing material is comparable to homoepitaxy GaN grown on bulk GaN crystals [34]. A downshift in the $\mathrm{D}^{0} \mathrm{X}$ peak of $5 \mathrm{meV}$ in the post with respect to the wing is due to an increase in tensile strain in the former.

\subsubsection{Silicon Substrates}

Absence of dislocations in the laterally overgrown material was also obtained for PE GaN films grown laterally and vertically from an unmasked, raised $\mathrm{GaN}$ stripe etched into a GaN/AlN/3C-SiC/Si(111) substrate, as shown by a representative sample in Figure 17 (a,b). The TEM image in Figure 17 (b) again reveals a significant reduction in density of vertically oriented threading dislocations in the laterally grown GaN region. Some horizontally oriented dislocations that do not propagate to the surface of the film were generated at the GaN/AIN interface of the GaN seed post. Dislocations generated at the GaN/AlN interface in the (0001) planes of the GaN stripes did not propagate vertically to the surface of the film.

\section{Summary}

Pendeo-epitaxy has been developed as an alternative and more simple approach of growing uniformly thin films with low densities of threading dislocations over the entire surface of a substrate. In particular, the growth of both discrete structures and coalesced $\mathrm{GaN}$ and $\mathrm{Al}_{\mathrm{x}} \mathrm{Ga}_{1-}$ ${ }_{\mathrm{x}} \mathrm{N}$ films and multilayer heterostructures using pendeoepitaxy on etched $\mathrm{GaN}$ seed layers previously grown on $\mathrm{AlN}(0001) / 6 \mathrm{H}-\mathrm{SiC}(0001)$ and $\mathrm{AlN}(0001) / \mathrm{SiC}(111) /$ $\mathrm{Si}(111)$ substrates has been demonstrated. Tilting in the coalesced $\mathrm{GaN}$ epilayers of $0.2^{\circ}$ was confined to areas 
of mask overgrowth; very little tilting was observed in the material suspended above the trenches or grown over unmasked stripes. The strong, low-temperature PL band-edge peak at $3.45 \mathrm{eV}$ with a FWHM of $<300 \mu \mathrm{eV}$ was measured in the overgrowth $\mathrm{GaN}$ material. The band-edge in the material on $\mathrm{Si}(111)$ was shifted by 10 $\mathrm{meV}$ to a lower energy, indicative of a greater tensile stress.

\section{ACKNOWLEDGMENTS}

The authors acknowledge Cree Research, Inc. for the $\mathrm{SiC}$ wafers. This work was supported by the Office of Naval Research under contracts N00014-98-1-0384 (Colin Wood, monitor) and N00014-98-1-0654 (John Zolper, monitor) as well as The Kenan Institute for Engineering, Technology and Science at NCSU. R.F. Davis was supported in part by the Kobe Steel, Ltd. Professorship. The authors also acknowledge the Georgia Technology Research Institute for use of their clean room facilities.

\section{REFERENCES}

[1] D. Kapolnek, S. Keller, R. Vetury, R.D. Underwood, P. Kozodoy, S.P. DenBaars, U.K. Mishra, Appl. Phys. Lett. 71, 1204-1206 (1997).

[2] Y. Kato, S. Kitamura, K. Hiramatsu, N. Sawaki, J. Cryst. Growth 144, 133 (1994).

[3] O. Nam, M. D. Bremser, B. L. Ward, R. J. Nemanich, R. F. Davis, Mater. Res. Soc. Symp. Proc. 449, 107 (1997).

[4] O Nam, MD Bremser, BL Ward, RJ Nemanich, RF Davis, Jpn. J. Appl. Phys. 36, L532 (1997).

[5] A Sakai, H Sunakawa, A Usui, Appl. Phys. Lett. 73, 481-483 (1998).

[6] H Marchand, XH Wu, JP Ibbetson, PT Fini, P Kozodoy, S Keller, JS Speck, SP DenBaars, UK Mishra, Appl. Phys. Lett. 73, 747-749 (1998).

[7] TS Zheleva, O-H Nam, MD Bremser, RF Davis, Appl. Phys. Lett. 71, 2472-2474 (1997).

[8] O-H Nam, MD Bremser, TS Zheleva, RF Davis, Appl. Phys. Lett. 71, 2638-2340 (1997).

[9] O-H Nam, T. S. Zheleva, MD Bremser, RF Davis, J. Electron. Mater. 27, 233 (1998).

[10] Zhonghai Yu , M.A.L. Johnson, T. Mcnulty, J.D. Brown, J.W. Cook,Jr, J.F. Schetzina, MRS Internet J. Nitride Semicond. Res. 3, 6 (1998).

[11] Y. Chen, R. Schneider, S. Y. Wang, R. S. Kern, C. H. Chen, C. P. Kuo, Appl. Phys. Lett. 75, 2062-2063 (1999).

[12] L. Jastrzebski, J. Cryst. Growth 63, 493 (1983).

[13] R. P. Gale, R. W. McClelland, J. C. C. Fan, C. O. Bozler, Appl. Phys. Lett. 41, 545-547 (1982).

[14] P. Vohl, C. O. Bozler, R. W. McClelland, A. Chu, A. J. Strauss, J. Cryst. Growth 56, 410-422 (1982).

[15] S Nakamura, M Senoh, S Nagahama, N Iwasa, T Yamada, T Matsushita, H Kiyoku, Y Sugimoto, T Kozaki, H Umemoto, M Sano, K Chocho, Appl. Phys. Lett. 72, 211213 (1998).
[16] S Nakamura, M Senoh, S Nagahama, N Iwasa, T Yamada, T Matsushita, H Kiyoku, Y Sugimoto, T Kozaki, H Umemoto, M Sano, K Chocho, Jpn. J. Appl. Phys. 37, L309 (1998).

[17] S. Nakamura, J. Cryst. Growth 201/202, 290-295 (1999).

[18] T. Zheleva, S. Smith, D. Thomson, K. Linthicum, T. Gerhke, P. Rajagopal, R. F. Davis, J. Electron. Mater. 28, L5L8 (1999).

[19] K. Linthicum, T. Gehrke, D. Thomson, E. Carlson, P. Rajagopal, T. Smith, D. Batchelor, R. F. Davis, Appl. Phys. Lett. 75, 196 (1999).

[20] T. Gehrke, K.J. Linthicum, D.B. Thomson, P. Rajagopal, A.D. Batchelor, R.F. Davis, MRS Internet J. Nitride Semicond. Res. 4S1, G3.2 (1999).

[21] K.J. Linthicum, T. Gehrke, D.B. Thomson, K.M. Tracy, E.P. Carlson, T.P. Smith, T.S. Zheleva, C.A. Zorman, M. Mehregany, R.F. Davis, MRS Internet J. Nitride Semicond. Res. 4S1, G4.9 (1999).

[22] D.B. Thomson, T. Gehrke, K.J. Linthicum, P. Rajagopal, R.F. Davis, MRS Internet J. Nitride Semicond. Res. 4S1, G3.37 (1999).

[23] Tsvetanka S. Zheleva, Scott A. Smith, Darren B. Thomson, Thomas Gehrke, Kevin J. Linthicum, Pradeep Rajagopal, Eric Carlson, Waeil M. Ashmawi, Robert F. Davis, MRS Internet J. Nitride Semicond. Res. 4S1, G3.38 (1999).

[24] T. Gehrke, K. J. Linthicum, P. Rajagopal et al, unpublished.

[25] R. F. Davis, T. Gehrke, K. J. Linthicum, T. S. Zheleva, E. A. Preble, P. Rajagopal, C. A. Zorman, M. Mehregany, $J$. Cryst. Growth 225, 134-140 (2001).

[26] S. Nakamura, M. Senoh, I. S. Nagahama, T. Matsushita, H. Kiyoku, Y. Sugimoto, T. Kozaki, H. Umemoto, M. Sano, T. Mukai, Jpn. J. Appl. Phys. 38, L226L229 (1999).

[27] T. Warren Weeks, Michael D. Bremser, K. Shawn Ailey, Eric Carlson, William G. Perry, Robert F. Davis, Appl. Phys. Lett. 67, 401-403 (1995).

[28] C. A. Zorman, A. J. Fleischman, A. S. Dewa, M. Mehregany, C. Jacob, S. Nishino, P. Pirouz, J. Appl. Phys. 78, 5136-5138 (1995).

[29] P. Fini, H. Marchand, J. P. Ibbetson, S. P. DenBaars, U. K. Mishra, J. S. Speck, J. Cryst. Growth 209, 581-590 (2000).

[30] S. Tomiya, K. Funato, T. Asatsuma, T. Hino, S. Kijima, T. Asano, M. Ikeda, Appl. Phys. Lett. 77, 636-638 (2000).

[31] P. Fini, A. Munkholm, C. Thompson, G. B. Stephenson, J. A. Eastman, M. V. Ramana Murty, O. Auciello, L. Zhao, S. P. DenBaars, J. S. Speck, Appl. Phys. Lett. 76, 3893 (2000).

[32] A. Sakai, H. Sunakawa, A. Kimura, A. Usui, Appl. Phys. Lett. 76, 442-444 (2000).

[33] Z. Liliental-Weber, M. Benamara, W. Swider, J. Washburn, J. Park, P.A. Grudowski, C.J. Eiting, R.D. Dupuis, MRS Internet J. Nitride Semicond. Res. 4S1, G4.6 (1999).

[34] C. Kirchner, V. Schwegler, F. Eberhard et al, Appl. Phys. Lett. 75, 1098-1100 (1999). 


\section{FIGURES}

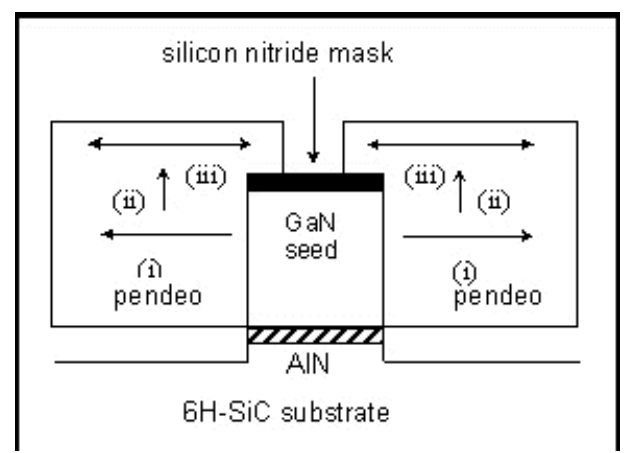

Figure 1. Schematic of pendeo-epitaxial growth of $\mathrm{GaN}$ from etched GaN ( $\left(\begin{array}{llll}1 & 1 & 2 & 0\end{array}\right)$ sidewalls and over a silicon nitride mask.

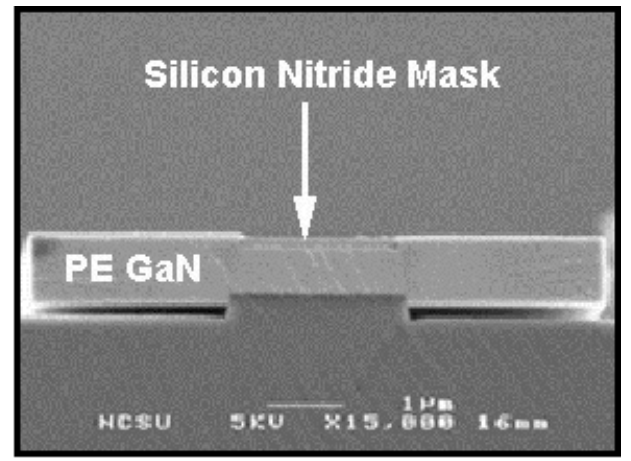

Figure 2. Cross-sectional SEM of a GaN pendeo-epitaxial growth structure with limited vertical growth from the seed sidewalls and no growth on the seed mask.

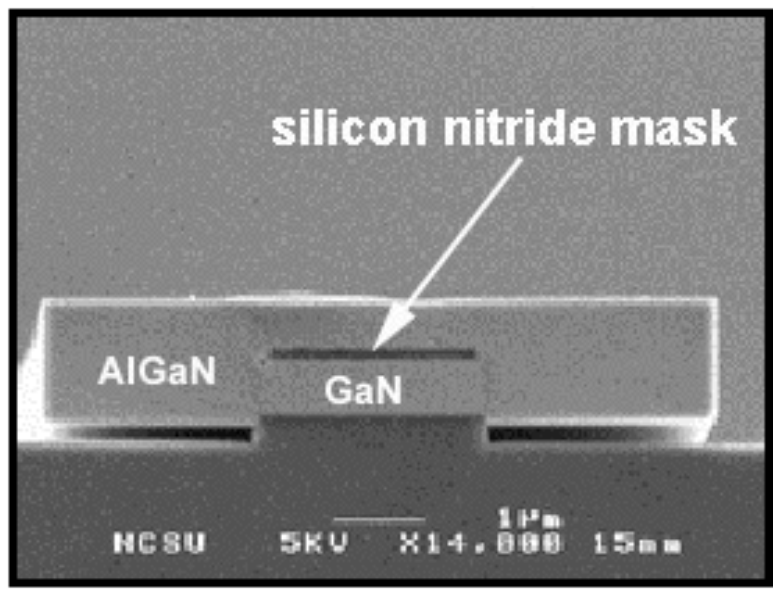

Figure 3. Cross-sectional SEM of a $\mathrm{GaN} / \mathrm{Al}_{10} \mathrm{Ga}_{90} \mathrm{~N}$ pendeo epitaxial growth structure showing coalescence over a seed mask.

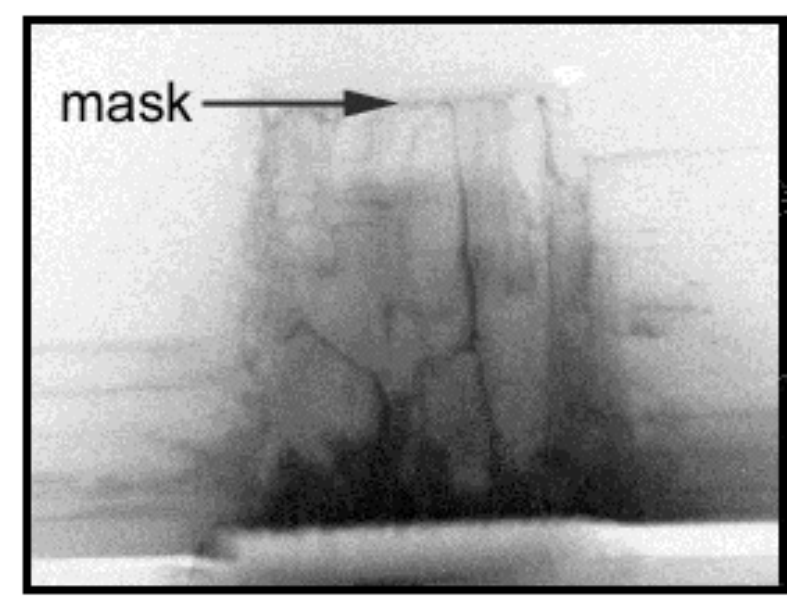

Figure 4. Cross-sectional TEM of a GaN pendeo-epitaxial structure showing confinement of threading dislocations under the seed mask, and a reduction of defects in the regrowth area.

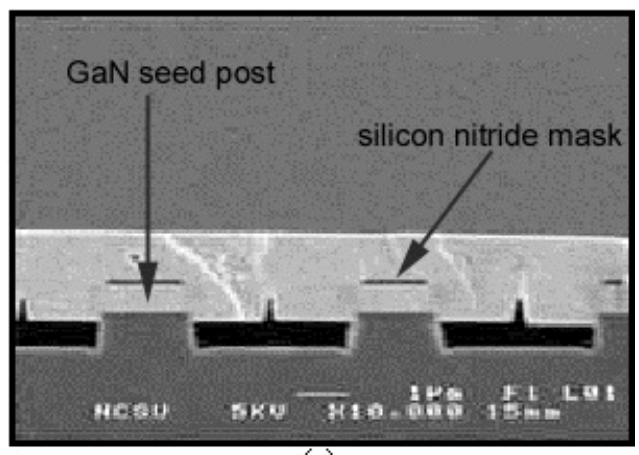

(a)

Figure 5a. Micrographs taken via cross-sectional SEM of examples of masked pendeo-epitaxial growth with coalescence over and between the seed forms resulting in a single $\mathrm{GaN}$ layer.

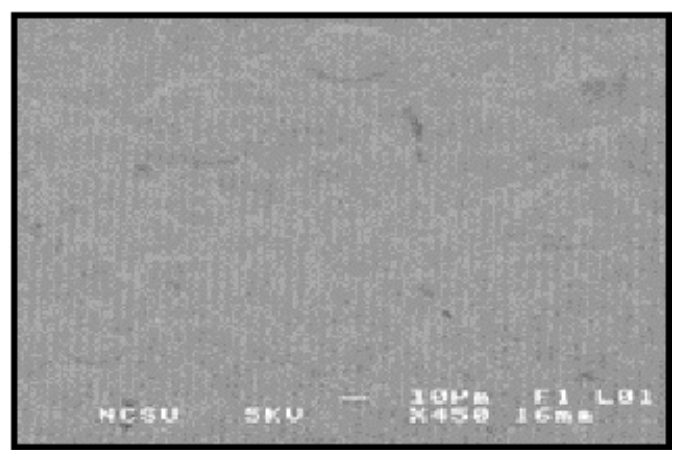

(b)

Figure 5b. Micrographs taken via plan-view SEM of examples of masked pendeo-epitaxial growth with coalescence over and between the seed forms resulting in a single $\mathrm{GaN}$ layer. 


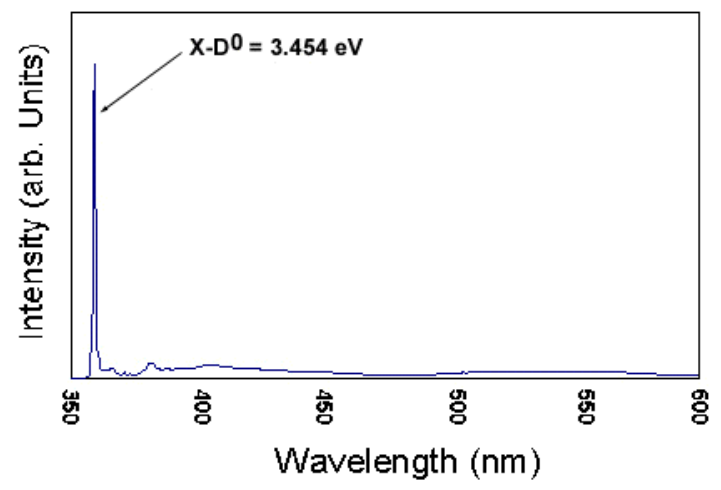

Figure 6. Low temperature $(7 \mathrm{~K})$ photoluminescence spectrum of a coalesced layer of pendeoepitaxial GaN grown on a GaN/ AlN/6H-SiC substrate.

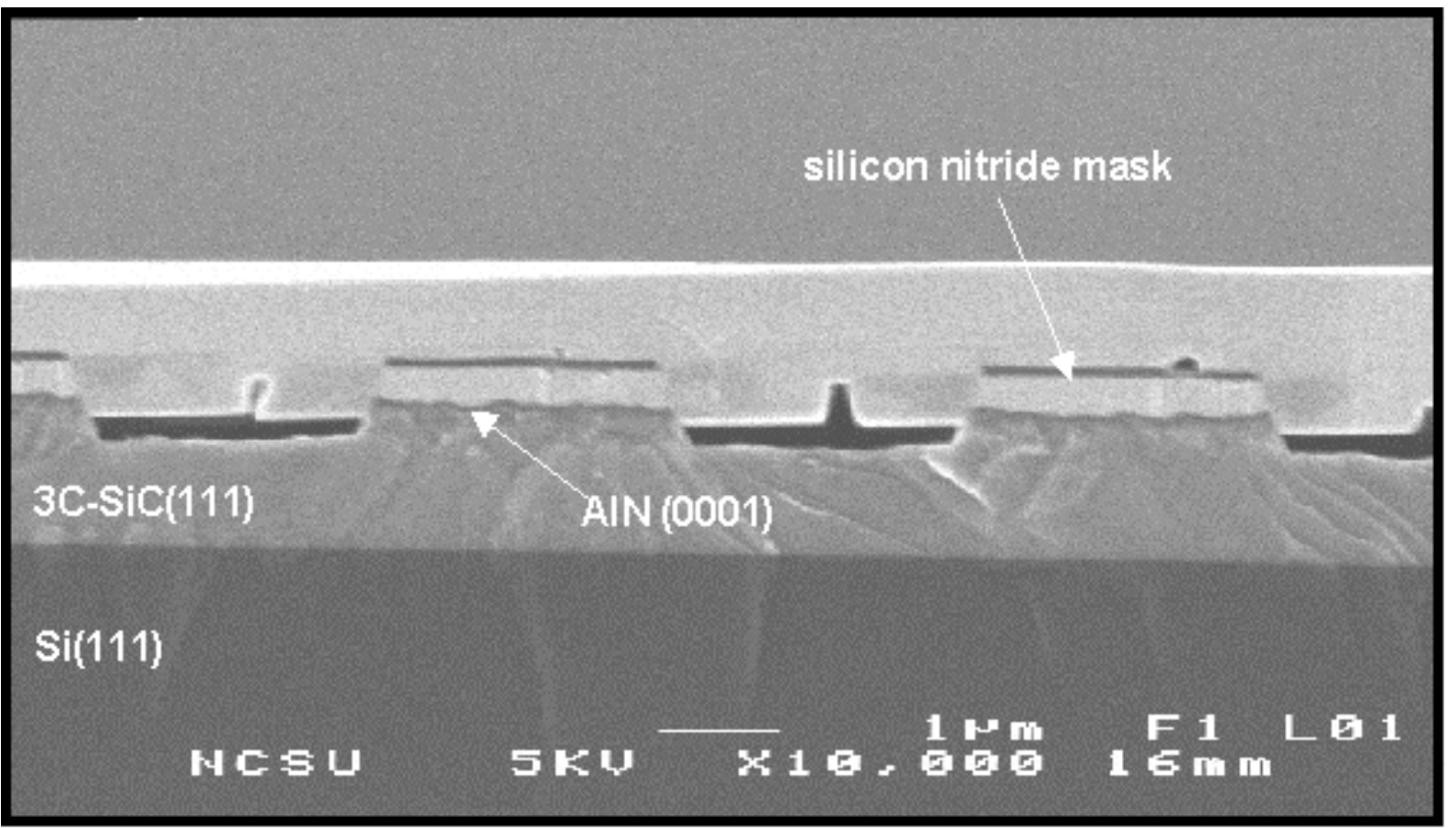

Figure 7. Cross-sectional SEM micrograph of a coalesced PE GaN epilayer deposited on a masked GaN/AlN/3C-SiC/Si(111) substrate.

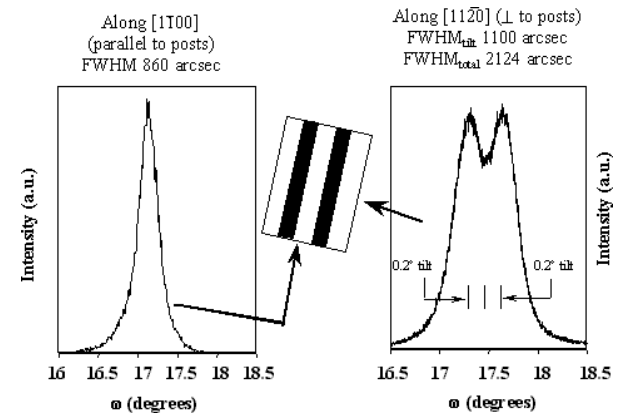

Figure 8. Double crystal XRD analysis of the tilting in the coalesced PE films. 


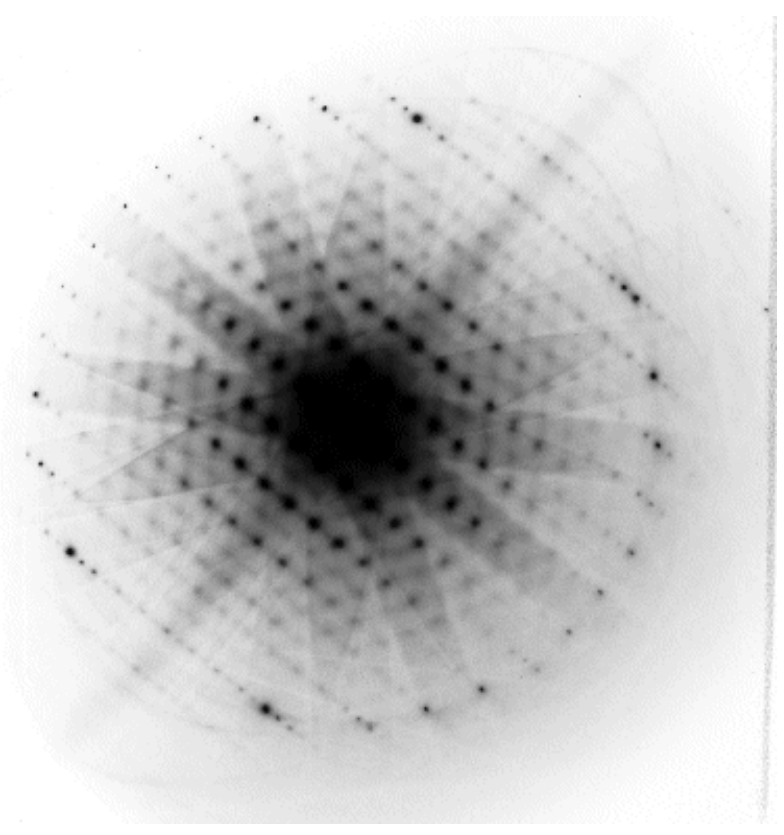

Figure 9a. TEM selected area diffraction patterns from (a) a small section of coalesced $\mathrm{PE} \mathrm{GaN}$ over a trench region and (b) a small area of PE GaN imaged from an area over the silicon nitride mask.

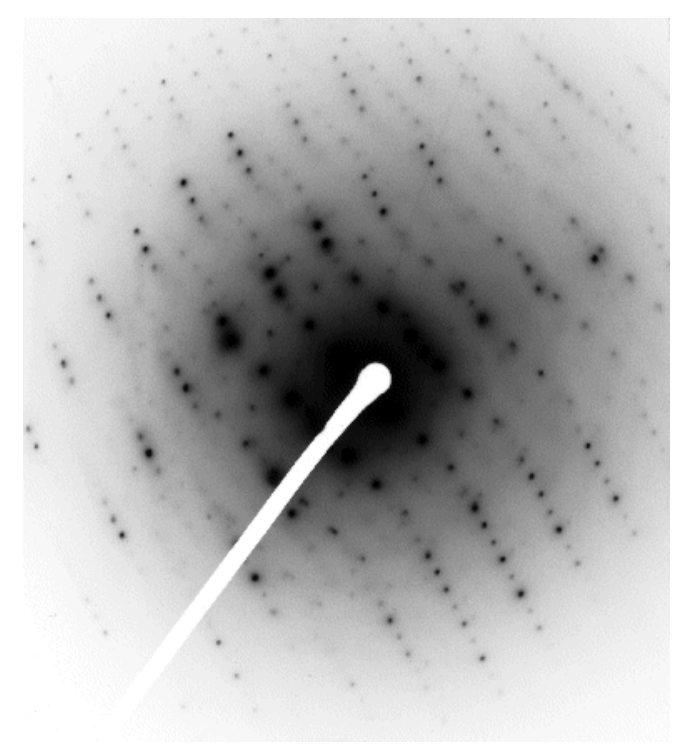

Figure 9b. see caption for Figure 9a

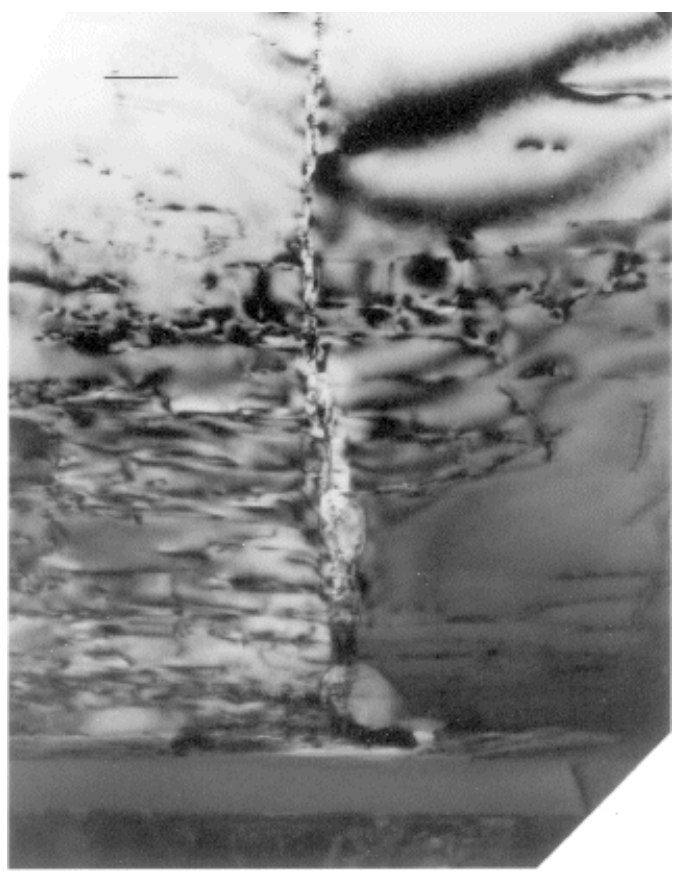

Figure 10. TEM micrograph of a GaN film grown over a silicon nitride mask. The coalescence boundary acted as a nucleation source for horizontally oriented dislocations.

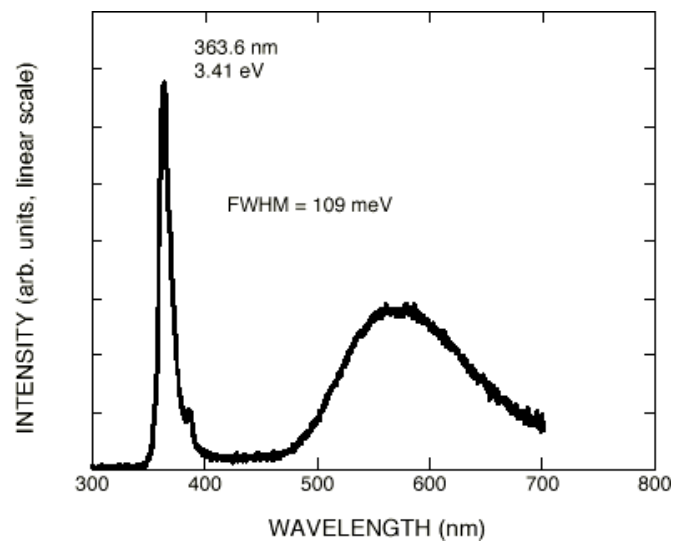

Figure 11. Room temperature photoluminescence of a coalesced layer of $\mathrm{PE} \mathrm{GaN}$ grown on a GaN/AlN/3C-SiC/ $\mathrm{Si}(111)$ substrate. 


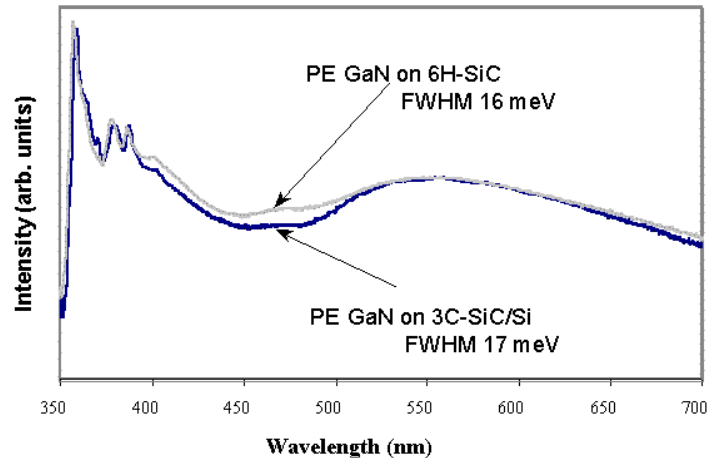

Figure 12. Comparison of low-temperature (14K) PL spectra of $\mathrm{PE} \mathrm{GaN}$ grown on GaN/AlN/6H-SiC and GaN/AlN/3C-SiC/ $\mathrm{Si}(111)$ substrates.

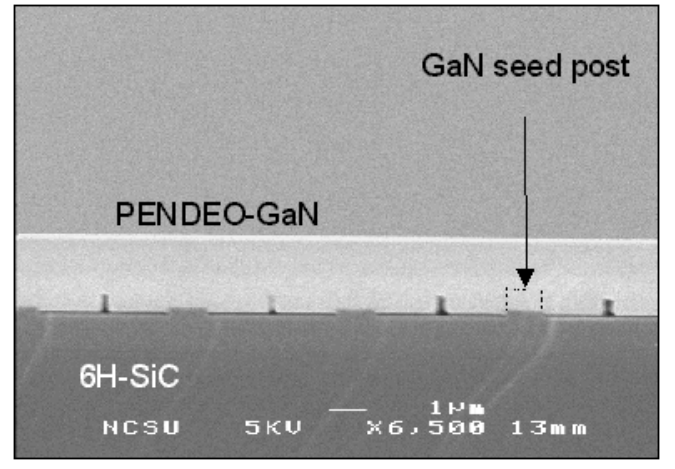

Figure 13a. GaN film grown on unmasked, submicron wide stripes of a GaN/AlN/6H-SiC(0001) substrate. No misregistry above the GaN stripes was observed.

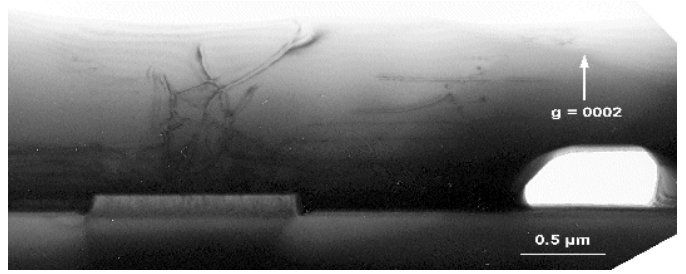

Figure 13b. TEM micrograph of the sample shown in (a). A significant reduction in the density of threading dislocations has been obtained in the PE GaN compared to the GaN stripes. 


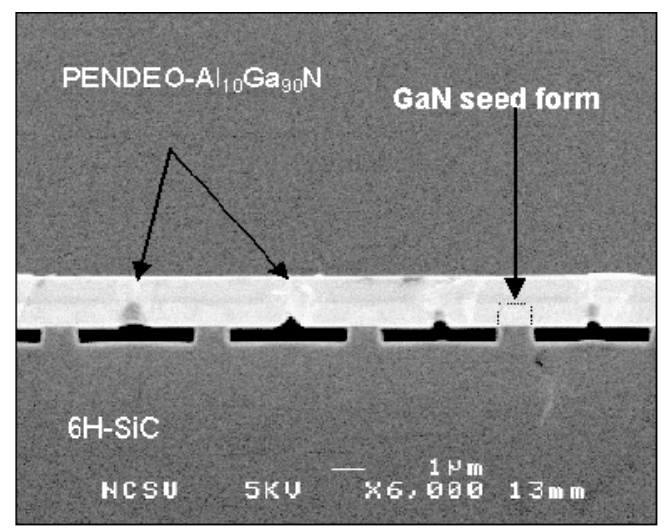

Figure $14 \mathrm{a} . \mathrm{Al}_{10} \mathrm{Ga}_{90} \mathrm{~N}$ film grown via pendeo-epitaxy on unmasked $\mathrm{GaN}$ seed posts on a $6 \mathrm{H}-\mathrm{SiC}$ substrate. No misregistry above the GaN seed posts is observable.

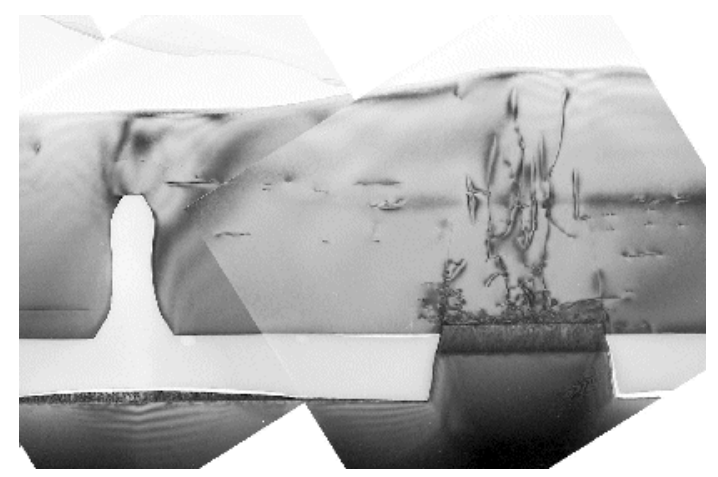

Figure 14b. TEM micrograph of the sample in (a). A significant reduction in density of threading dislocations in the pendeoepitaxial $\mathrm{Al}_{10} \mathrm{Ga}_{90} \mathrm{~N}$ compare to the $\mathrm{GaN}$ seed post is obvious.

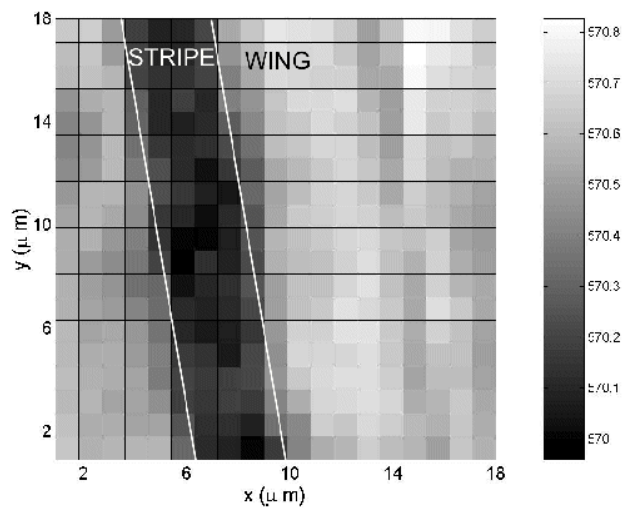

Figure 15. Two-dimensional space plot of Raman line frequency of an uncoalesced PE GaN film (specifically a wing and stripe region). An increase in the line frequency represents a relaxation of strain in the material. 


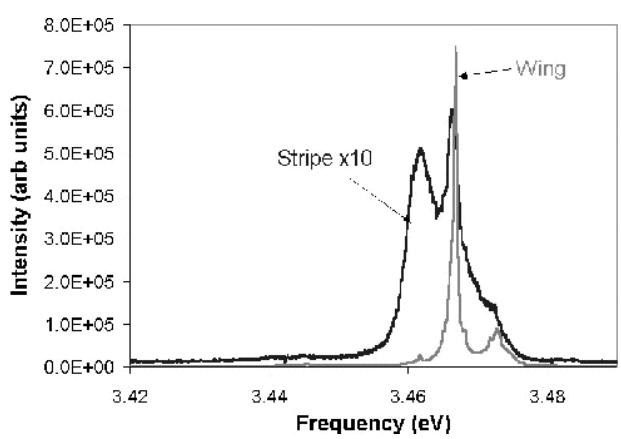

Figure 16. Photoluminescence (PL) spectra taken from the wing and stripe region of an uncoalesced PE GaN film. Note the FWHM value for the wing peak is $<300 \mu \mathrm{eV}$.

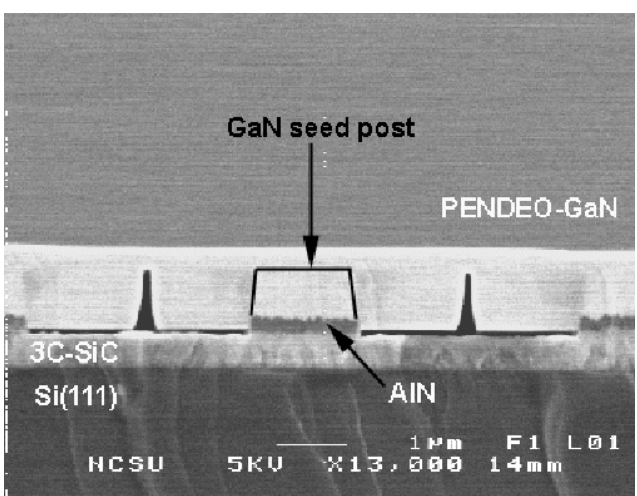

Figure 17a. Coalesced GaN films grown on un-masked GaN stripes on a GaN/AlN/3C-SiC/Si(111) substrate. No misregistry above the $\mathrm{GaN}$ seed post was observed.

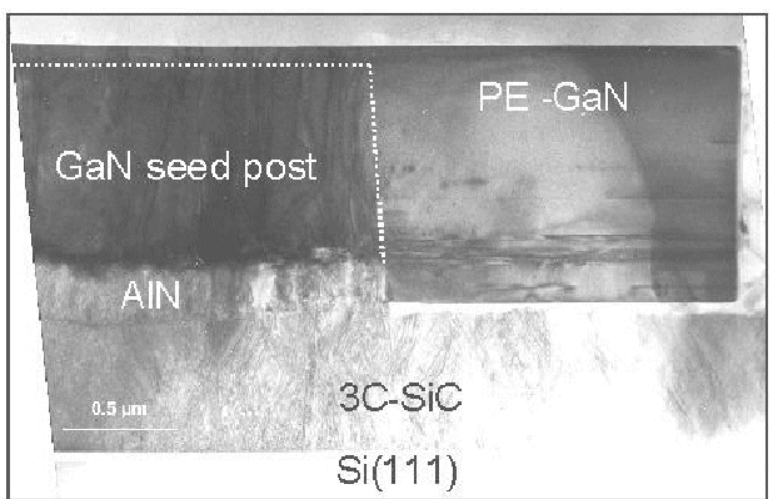

Figure 17b. TEM micrograph of the sample in (a). A significant reduction in density of threading dislocations occurred in the pendeo-epitaxial GaN compared to the GaN stripes. 